\title{
Psychiatry and neurodevelopmental disorders: experts by experience, clinical care and research
}

\author{
Regi T. Alexander, Peter E. Langdon, Jean O'Hara, Andreana Howell, Tadhgh Lane, Reena Tharian and \\ Rohit Shankar
}

\begin{abstract}
Summary
People with neurodevelopmental disorders often present with challenging behaviours and psychiatric illnesses. Diagnosis and treatment require patients, families and healthcare professionals to work closely together in partnership, acknowledging their respective areas of expertise. Good treatment outcomes should also be underpinned by robust research evidence. Key research priorities are highlighted.
\end{abstract}

\section{Keywords}

Neurodevelopmental disorders; intellectual disability; autism; attention-deficit hyperactivity disorder; experts by experience.

\section{Copyright and usage}

(C) The Authors, 2020. Published by Cambridge University Press on behalf of the Royal College of Psychiatrists.
Regi T. Alexander is a consultant psychiatrist in Hertfordshire Partnership University NHS Foundation Trust, UK, visiting professor at the University of Hertfordshire, UK, co-convenor of the Research in Developmental Neuropsychiatry (RADiANT) network, and President of the Royal Society of Medicine's Intellectual Disability Forum. He is also a cardiac arrest survivor. Peter E. Langdon is a consultant clinical and forensic psychologist in Coventry and Warwickshire Partnership NHS Trust, UK, trustee of Asperger East Anglia, co-convenor of the RADIANT network, and professor, Centre for Educationa Development, Appraisal and Research, University of Warwick, UK. Jean O'Hara is a consultant psychiatrist in South London and Maudsley NHS Foundation Trust, UK, visiting senior lecturer at Institute of Psychiatry, Psychology and Neurosciences, Kings College London and former National Clinical Director for Learning Disabilities at NHS England. Andreana Howell is an advisor to the RADIANT network, hosted by Hertfordshire Partnership University NHS Foundation Trust, UK. She is also the mother of an adult daughter with Down syndrome. Tadhgh Lane is an advisor to the RADiANT network, hosted by Hertfordshire Partnership University NHS Foundation Trust, UK. He has a twin brother with autism, mental health difficulties and epilepsy. Reena Tharian is currently a clinical pharmacist in Norfolk \& Suffolk NHS Foundation Trust, UK, with a specialist interest in the appropriate use of psychotropic medication in people with neurodevelopmental disorders, and was previously a community pharmacist for several years. She is also a cancer survivor. Rohit Shankar is a consultant neuropsychiatrist in Cornwall NHS Foundation Trust, Director of Cornwall Intellectual Disability and Epilepsy Research (CIDER) Centre, Research Lead of the RADIANT network and honorary associate professor at Exeter Medical School. Winner of several BMJ awards, he was awarded the MBE in 2016.

Neurodevelopmental disorders (NDDs) are conditions that are grouped together because they are characterised by some form of disruption to typical brain development. This definition would mean that the range of conditions that are included in this group would be very wide and disparate. In an approach that provides much greater clinical utility for patients and professionals, the DSM-5 only includes intellectual disabilities, autism spectrum disorder (ASD), attention-deficit hyperactivity disorder (ADHD), communication disorders, specific learning disorders and motor disorders as part of this group. Although heterogeneous, they share some common characteristics: childhood onset, a steady rather than remitting and relapsing course, early onset of neurocognitive deficits, high heritability indices and a marked overlap between their core symptom domains. ${ }^{1}$ Although their lifetime prevalence varies (1\% for ASD, $2 \%$ for intellectual disability, $2.5 \%$ for ADHD, etc), NDDs often co-occur, and up to $10 \%$ of the population may have one, according to a recently formed coalition of charities in this field (https://embracingcomplexity.org.uk). They point out that this often causes problems in accessing help, particularly in countries where services are set up exclusively for one disorder and exclude people because they have other co-occurring conditions.
The diagnosis and treatment of mental health difficulties in people with NDDs sometimes raises contentious issues. In this editorial, we explore some of the key themes, starting with the interface between 'challenging behaviour' and psychiatric conditions. We then discuss the central role of patients and their families (i.e. experts with lived experience) in the treatment process, and how that involvement should complement the expertise of health professionals (i.e. experts by profession) if good outcomes are to be achieved. Finally, we will reflect on how high-quality research is central to the delivery of good treatment outcomes, and set out some of the key research themes that are currently prioritised.

\section{Challenging behaviour and psychiatric conditions}

Compared with the general population, people with NDDs can have higher rates of challenging behaviours. This may bring them into contact with mental health professionals. A socially constructed and descriptive concept often driven by environmental factors, challenging behaviour is certainly not a psychiatric diagnosis. However, it is worth noting that compared with the general population, people with NDDs also have higher rates of psychiatric morbidity. Some of this psychiatric morbidity may well present with challenging behaviours. Indeed, the presence of an NDD, and the communication difficulties associated with it, will affect the way in which psychiatric morbidity manifests itself, the modes of treatment and the outcomes of treatment. This means that when an individual with an NDD presents with challenging behaviour, a careful assessment for any coexisting psychiatric or physical health conditions is required. For example, the aetiology of challenging behaviour displayed by a previously welladjusted, middle-aged man with Down syndrome could be underlying physical health concerns (pain, sensory visual or auditory problems, constipation, sleep issues, undiagnosed hypothyroidism), psychological issues (depressive or anxiety features, bereavement), cognitive concerns (early onset of dementia), environmental stressors or a combination of them all. It is this complexity that makes the diagnosis and treatment of mental and behavioural disorders in people with NDDs a particularly challenging area of psychiatric practice. ${ }^{2}$

\section{Experts with lived experience and experts by profession}

In such situations, it is important to centre the lived experience of patients and their families within all clinical care, and thereby 
Table 1 Recommended research priorities in Autism (NICE Clinical Guideline 142 https://www.nice.org.uk/guidance/cg142), ADHD (NICE Clinical Guideline 87 https://www.nice.org.uk/guidance/ng87), Challenging behaviour and learning disabilities (NICE Clinical Guideline 11 https://www.nice.org. uk/guidance/ng11) and Mental health problems in people with learning disabilities (NICE Clinical Guideline 54 https://www.nice.org.uk/guidance/ng54)

$\begin{array}{ll}\text { Neurodevelopmental disorder } & \text { Research priorities } \\ \text { Autism } & \text { 1. A key worker approach for children and young people with autism and their families } \\ & \text { 2. Managing challenging behaviour in children and young people with autism } \\ & \text { 3. Managing sleep problems in children with autism }\end{array}$

$\mathrm{ADHD}$
1. Brief, group-based, ADHD-focused, parent-training intervention in children and young people aged $5-18$ years

2. Medication choice in people with coexisting conditions

3. Medication choice in people with no previous medication for ADHD

4. Prescribing beyond monotherapy
Intellectual disability (Learning disability) and challenging
behaviour
Intellectual disability (Learning disability) and mental
health problems

Intellectual disability (Learning disability) and challeng
behaviour
Intellectual disability (Learning disability) and mental
health problems

Intellectual disability (Learning disability) and challeng
behaviour
Intellectual disability (Learning disability) and mental
health problems

Intellectual disability (Learning disability) and challeng
behaviour
Intellectual disability (Learning disability) and mental
health problems

1. Preventing challenging behaviour from developing in children aged $<5$ years with intellectual disability

2. Interventions to reduce the frequency and extent of moderate-to-severe challenging behaviour in community settings

3. Locally accessible care

4. Factors associated with sustained, high-quality residential care
1. Develop case identification tools for common mental health problems

2. Psychological interventions for children and young people with internalising disorders

3. Psychological interventions for depression and anxiety disorders in adults with mild-to-moderate intellectual disability

4. Pharmacological and psychological interventions for anxiety disorders in people with intellectual disability who have autism

5. Psychosocial interventions for people with severe and profound intellectual disability

6. The experiences of people with intellectual disability and mental health problems in services

ADHD, attention-deficit hyperactivity disorder.

minimise the risk of 'diagnostic overshadowing', where the presentation is inappropriately explained away by the presence of an NDD. From embedding reasonable adjustments to ensure psychiatric services are accessible, to providing treatments that are developmentally appropriate and diligently avoiding restrictive treatment strategies such as overmedication with psychotropics or inappropriate admission to psychiatric hospital, patients and their families (experts with lived experience) have an important role to play as partners in mental healthcare. ${ }^{2}$ In this regard, it is also important to appreciate that the risk is not merely one of restrictive treatments. There is the equally unacceptable risk that by formulating everything solely through the prism of lived experience, treatable psychiatric conditions may potentially be missed or undertreated. This may well be because healthcare professionals come under pressure to avoid the diagnosis of psychiatric illness because of an erroneous assumption that the act of diagnosis itself is one of labelling that leads to restrictive practice. When this happens, the risk of excess morbidity and mortality in people with NDDs can get worse.

To elaborate on this, some of the healthcare professionals who are authors of this editorial draw upon their personal experiences with major physical illnesses. The experience of having had multiple cardiac arrests and heart failure has made one an expert by lived experience, who can give meaningful advice on improving the patient experience in cardiac intensive care units, while simultaneously acknowledging that deciding the right treatment for cardiac failure does require a different kind of specialist professional expertise. Likewise, living through an invasive cancer has allowed another to become an expert on the patient experience of the side-effects of chemotherapy, while also recognising that this expertise is qualitatively different from the specialist knowledge of an oncologist. Yet another, who developed problems with chronic pain, can easily articulate the overwhelming and enduring feelings of pain so severe that it was impossible to get out of bed, along with the side-effects of the numerous powerful medications that were prescribed, until collaboratively, using the expert knowledge of pain specialists, the most helpful medication was found, leading to improvements in quality of life. We surmise from this that the patient is indeed the expert with lived experience of the condition; appropriately qualified professionals are the experts in terms of the science that underpins diagnosis and treatments, and the two must listen and learn from each other. Some say that 'the real expert is always the patient', and this is true, but both professionals and patients must avoid positioning themselves upon a polarised continuum. Good care occurs when professionals recognise patient expertise. Recognition of professional knowledge in diagnosis and treatment is an equally important component of good care, and one that will avoid disadvantaging some patient groups. Just as one would never expect that disadvantage in cancer or cardiac failure treatment, one should not expect it in the field of NDDs and mental health conditions. This is not to say that patients and families of people with NDD do not have any expertise in diagnosis or treatment - far from it. Indeed, one of the expert by experience authors can draw upon his own experience to demonstrate this point well. There was a situation when he and the rest of the family were quite convinced that their loved one's challenging behaviour was driven in part by an underlying mental illness. They knew this because they were the people who were best placed to observe and appreciate the nuances of the family member's clinical presentation. This message was not accepted by some professionals. It took the family's dogged perseverance, a 
new team of professionals committed to working collaboratively with them, the safe space of an in-patient unit and a systematic assessment to identify the complex diagnostic strands and secure the best treatment outcomes. Likewise, another expert by experience has written about how he carefully and perceptively recorded his daughter's clinical presentation as suggestive of depression, but was not listened to appropriately by professionals for quite some time. This experience later led to co-produced guidelines on improving family experiences of accessing and engaging with mental health/NDD services. ${ }^{2}$ Suffice to say that if we want equity of treatment outcomes in NDDs as much as equity of access to healthcare, then healthcare professionals, patients and family members should work collaboratively, paying due attention to their respective areas of expertise. In that way, the lived experience of patients and families can effectively and safely complement treatment approaches that are informed by scientific knowledge and expertise. In this whole process, the importance of professionals sharing information in an accessible format with patients and their carers cannot be overemphasised.

\section{Good treatment outcomes and research}

Good treatment outcomes for patients can be achieved only by a good evidence base and high-quality research. For decades, this area has been hampered by a relative paucity in relation to basic sciences, clinical practice and service models. This is changing gradually, and in the past 6 years there have been a number of National Institute of Healthcare and Care Excellence (NICE) clinical guidelines on NDDs, a development indicating a growing evidence base in this area. Key research priorities for people with intellectual disability, ASD and ADHD, as drawn from the respective NICE guidelines, are summarised in Table 1.

More recently, a themed review published by the National Institute of Health Research (NIHR) Dissemination Centre highlighted 23 NIHR-funded influential studies, many co-produced by people with intellectual disability and family carers, strengthening our point about the importance of professionals and those with lived experience working collaboratively. It set out future research priorities that included health checks, intellectual disability services for children, mental well-being promotion, mental health self-management programmes, supported living outside hospital settings, training regarding domestic abuse of women with intellectual disability and reasonable adjustments in general hospitals. ${ }^{3}$ There is also a need to use routinely collected clinical data in a meaningful way, to measure treatment outcomes and benchmark services.

In addition to these, there have been several other promising new areas of research. Genetics is not only providing an understanding of neurodevelopmental conditions, but is also looking at developing interventional tools. ${ }^{4}$ Although pharmacogenomics ${ }^{5}$ is looking to establish itself in giving insights to medication response based on unique individual characteristics, efforts to identify and validate biomarkers in various domains are under way. Elsewhere, there are enquires into issues such as diet types and the human microbiome influence on brain maturation. Technological advances in improving diagnosis, providing bio-feedback responses and engaging individuals into vocations are being piloted. These person-centred approaches for people with neurodevelopmental conditions offer the hope that psychiatric and behavioural comorbidity in this group can be addressed through innovative means. In the quest to explore these new frontiers, it is important not to lose sight of those simple and effective interventions such as annual health checks, which have proved to make a positive difference.

Regi T. Alexander (D), Hertfordshire Partnership University NHS Foundation Trust, UK; Peter E. Langdon (1), Coventry and Warwickshire Partnership NHS Trust, UK ; Jean O'Hara, South London and Maudsley NHS Foundation Trust, UK;

Andreana Howell, RADIANT network, Hertfordshire Partnership University NHS

Foundation Trust, UK; Tadhgh Lane, RADIANT network, Hertfordshire Partnership

University NHS Foundation Trust, UK: Reena Tharian, Norfolk \& Suffolk NHS Foundation

Trust, UK; Rohit Shankar (D), Cornwall NHS Foundation Trust, UK

Correspondence: Regi T. Alexander. Email: regialexander@nhs.net

First received 2 Nov 2020, final revision 13 Nov 2020, accepted 16 Nov 2020

\section{Author contributions}

The first draft was written by R.T.A. based on opinions and suggestions provided by P.E.L., J.O.H., A.H., T.L., R.T. and R.S. All authors reviewed and commented on the draft and contributed equally to the final version.

\section{Declaration of interest}

None of the authors have any interests to declare in relation to this study. R.T.A., P.E.L. and J. $\mathrm{H}$. are editors of this special issue, but are not involved in the peer review of this editorial. R.S. has received institutional and research support and personal fees from Union Chimique Belge (UCB), Eisai, Bial, Veriton Pharma, GW Pharma, Livanova and Desitin outside the submitted work.

\section{References}

1 Thapar A, Cooper M, Rutter M. Neurodevelopmental disorders. Lancet Psychiatry 2017; 4: 339-46.

2 Chester V, James N, Rogers I, Grace J, Alexander RT. Mental health problems in people with intellectual or developmental disabilities: the involvement of family and carers. In Oxford Textbook of Psychiatry of Intellectual Disability (eds S Bhaumik, Alexander RT): 265-274. Oxford University Press, 2020.

3 National Institute for Health Research (NIHR) Dissemination Centre. Better Health and Care for All. NIHR, 2020 (https://content.nihr.ac.uk/nihrdc/themedreview-04326-BCAHFA/Better-Health_Care-For-FINALWEB.pdf).

4 Ilaria P, Luis GR, Hanna S, Gaia N. Neurodevelopmental disorders: from genetics to functional pathways. Trends Neurosci 2020; 43: 608-21.

5 Cacabelos R. Pharmacogenomics of drugs used to treat brain disorders. Expert Rev Precision Med Drug Dev 2020; 5(3): 181-234. 